Supplement of Atmos. Chem. Phys., 19, 4193-4210, 2019

https://doi.org/10.5194/acp-19-4193-2019-supplement

(C) Author(s) 2019. This work is distributed under

the Creative Commons Attribution 4.0 License.

(c) (i)

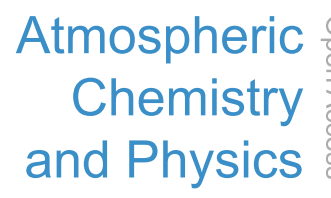

Supplement of

\title{
Contrasting local and long-range-transported warm ice-nucleating particles during an atmospheric river in coastal California, USA
}

Andrew C. Martin et al.

Correspondence to: Kimberly Prather (kprather@ucsd.edu)

The copyright of individual parts of the supplement might differ from the CC BY 4.0 License. 


\section{Supplemental ATOFMS Analysis of Precipitation Methods}

The size-resolved single particle mixing state of precipitation sample residues was measured using an ATOFMS (E. Gard et al., 1997). Samples were aerosolized using a collision-style nebulizer and dried using two silica diffusion driers. Samples were stirred using a magnetic stir bar to ensure that insoluble particles had not settled and were being aerosolized. Temporally adjacent precipitation samples were combined to obtain the requisite sample volume for use with the atomizer $(75-100 \mathrm{~mL})$, but samples were not combined across kinematic period boundaries. Single particle spectra and size data were imported into Matlab (The MathWorks, Inc.) and analyzed via the software toolkit FATES (Sultana, Cornwell, et al., 2017). Particles were divided into clusters based on their mass spectral features via an adaptive neural network and recombined based upon their characteristic mass spectra and size distributions (Rebotier \& Prather, 2007; Song et al., 1999). Particles were separated into 4 different classes based upon previous ATOFMS lab and field studies: biomass burning (BB), organic carbon (OC), dust, and biological particles (Bio). Table SM1 below shows the representative peaks and literature references associated with each particle type.

Table S1: Insoluble residue particle types identified using ATOFMS/FATES.

\section{Particle type}

\begin{tabular}{|c|c|}
\hline $\begin{array}{l}\text { Biomass burning } \\
\text { (BB) }\end{array}$ & $\begin{array}{l}\text { Intense }{ }^{39} \mathrm{~K}^{+} \text {and }{ }^{97} \mathrm{HSO}_{4}^{-} \text {, minor contributions from }{ }^{12} \mathrm{C}^{+},{ }^{26} \mathrm{CN}^{-},{ }^{46} \mathrm{NO}_{2}{ }^{-} \\
{ }^{62} \mathrm{NO}_{3}^{-},{ }^{125} \mathrm{H}_{(}\left(\mathrm{NO}_{3}\right){ }_{2}^{-} \text {(P. J. Silva et al., 1999). }\end{array}$ \\
\hline $\begin{array}{l}\text { Organic carbon } \\
\qquad(\mathrm{OC})\end{array}$ & $\begin{array}{l}{ }^{27} \mathrm{C}_{2} \mathrm{H}_{3}{ }^{+},{ }^{29} \mathrm{C}_{2} \mathrm{H} 5,{ }^{37} \mathrm{C}_{3} \mathrm{H}^{+},{ }^{43} \mathrm{CH}_{3} \mathrm{CO}^{+} / \mathrm{CHNO}^{+} \text {(Qin et al., 2012; P. Silva } \\
\text { \& Prather, 2000; Spencer \& Prather, 2006). }\end{array}$ \\
\hline Dust & $\begin{array}{l}{ }^{7} \mathrm{Li}^{+},{ }^{27} \mathrm{Al}^{+},{ }^{39} \mathrm{~K}^{+},{ }^{48,64} \mathrm{Ti} \mathrm{TiO}^{+},{ }^{54,56} \mathrm{Fe}^{+},{ }^{60} \mathrm{SiO}_{2}{ }^{-76} \mathrm{SiO}_{3}^{-},{ }^{79} \mathrm{PO}_{3}^{-} \text {(P. J. Silva } \\
\text { et al., 2000). }\end{array}$ \\
\hline Biological (Bio) & $\begin{array}{l}\text { Strong }{ }^{39} \mathrm{~K}^{+} \text {and }{ }^{79} \mathrm{PO}_{3}^{-} \text {, minor contributions from organic fragments } \\
\text { (Fergenson et al., 2004; Sultana, Al-Mashat, et al., 2017). }\end{array}$ \\
\hline
\end{tabular}

2. Supplemental ATOFMS Analysis of Precipitation Results

See Discussion in Main Document, section 4.4. 


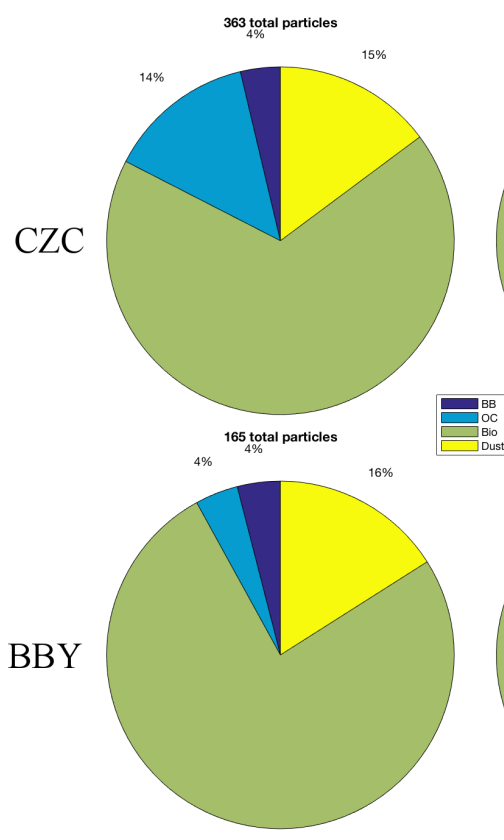

Early AR

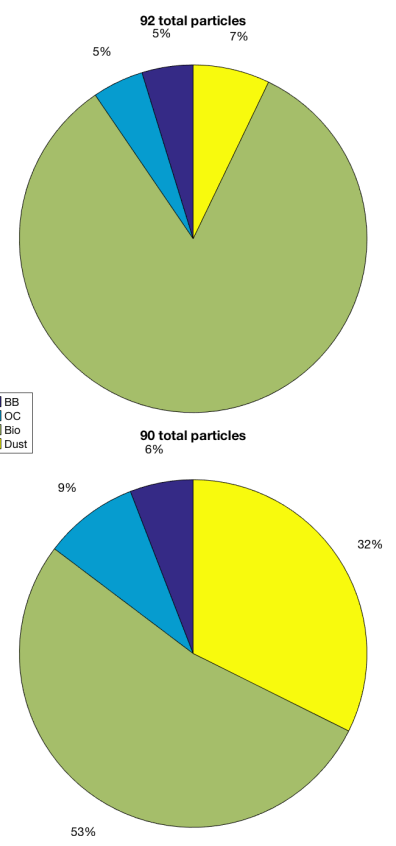

Barrier Jet

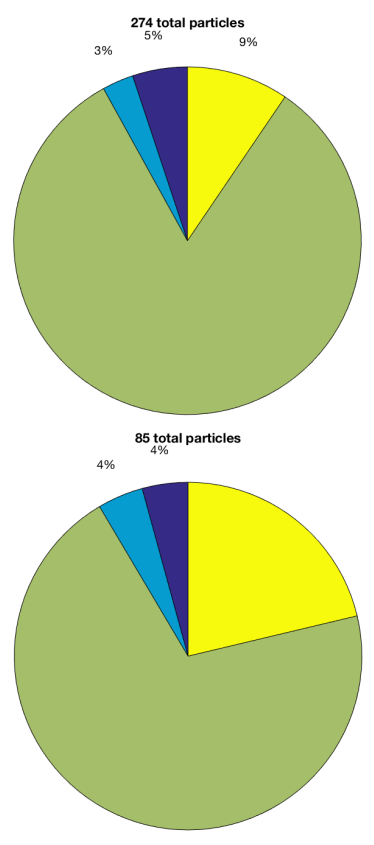

Peak AR

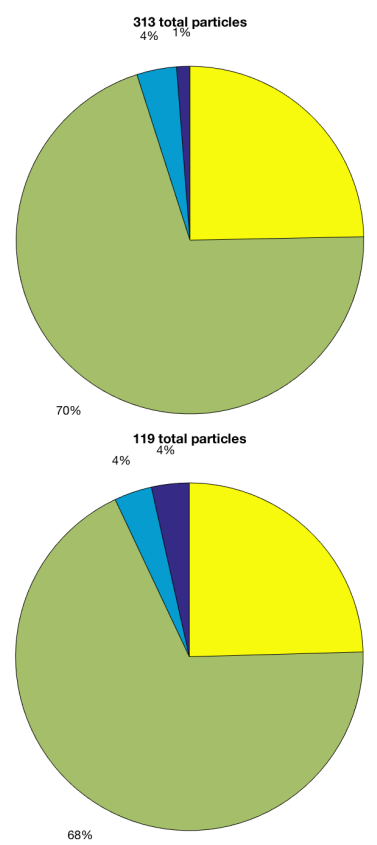

Post CF

Figure S1: Particle fraction by ATOFMS/FATES detected and classified type at CZC (top row) and BBY (bottom row) during precipitation collected during the kinematic periods: Early AR, Barrier Jet, Peak AR, and Post CF.

\section{References}

Fergenson, D. P., Pitesky, M. E., Tobias, H. J., Steele, P. T., Czerwieniec, G. A., Russell, S. C., et al. (2004). Reagentless Detection and Classification of Individual Bioaerosol Particles in Seconds. Analytical Chemistry, 76(2), 373-378. https://doi.org/10.1021/ac034467e

Gard, E., Mayer, J. E., Morrical, B. D., Dienes, T., Fergenson, D. P., \& Prather, K. A. (1997).

Real-Time Analysis of Individual Atmospheric Aerosol Particles: Design and Performance of a Portable ATOFMS. Analytical Chemistry, 69(20), 4083-4091. https://doi.org/10.1021/ac970540n

Qin, X., Pratt, K. a., Shields, L. G., Toner, S. M., \& Prather, K. a. (2012). Seasonal comparisons of single-particle chemical mixing state in Riverside, CA. Atmospheric Environment, 59, 587-596. https://doi.org/10.1016/j.atmosenv.2012.05.032

Rebotier, T. P., \& Prather, K. A. (2007). Aerosol time-of-flight mass spectrometry data analysis: A benchmark of clustering algorithms. Analytica Chimica Acta, 585, 38-54. https://doi.org/10.1016/j.aca.2006.12.009

Silva, P., \& Prather, K. (2000). Interpretation of mass spectra from organic compounds in aerosol time-of-flight mass spectrometry. Analytical Chemistry, 72(15), 3553-62. Retrieved from http://www.ncbi.nlm.nih.gov/pubmed/10952542

Silva, P. J., Liu, D., Noble, C. A., \& Prather, K. A. (1999). Size and Chemical Characterization of Individual Particles Resulting from Biomass Burning of Local Southern California Species. Environmental Science \& Technology, 33(18), 3068-3076.

Silva, P. J., Carlin, R. a, \& Prather, K. a. (2000). Single particle analysis of suspended soil dust 
from Southern California. Atmospheric Environment, 34(11), 1811-1820.

https://doi.org/10.1016/S1352-2310(99)00338-6

Song, X. H., Hopke, P. K., Fergenson, D. P., \& Prather, K. a. (1999). Classification of single particles analyzed by ATOFMS using an artificial neural network, ART-2A. Analytical Chemistry, 71(4), 860-865. https://doi.org/10.1021/ac9809682

Spencer, M. T., \& Prather, K. a. (2006). Using ATOFMS to Determine OC/EC Mass Fractions in Particles. Aerosol Science and Technology, 40, 585-594. https://doi.org/10.1080/02786820600729138

Sultana, C. M., Al-Mashat, H., \& Prather, K. A. (2017). Expanding Single Particle Mass Spectrometer Analyses for the Identification of Microbe Signatures in Sea Spray Aerosol. Analytical Chemistry, 89(19), 10162-10170. https://doi.org/10.1021/acs.analchem.7b00933

Sultana, C. M., Cornwell, G. C., Rodriguez, P., \& Prather, K. A. (2017). FATES: a flexible analysis toolkit for the exploration of single-particle mass spectrometer data. Atmospheric Measurement Techniques, 10(4), 1323-1334. https://doi.org/10.5194/amt-10-1323-2017 